\title{
POSITRON ANNIHILATION STUDY OF STRUCTURAL RELAXATION IN AMORPHOUS
} $\mathrm{Fe}_{40} \mathrm{Ni}_{40} \mathrm{~B}_{20}$ ALLOY

\author{
J. Pavlovský \\ Institute of Physical Metallurgy, Czecho-Slovak Academy of Sciences, Brno, \\ Czecho-Slovakia \\ (Received December 27, 1990)
}

\begin{abstract}
Structural relaxation of amorphous $\mathrm{Fe}_{40} \mathrm{Ni}_{40} \mathrm{~B}_{20}$ alloy has been studied by the method of angular correlation of positron annihilation. Inexpressive changes of the spectra after the annealing for $2 \times 10^{3}$ min at $473 \mathrm{~K}$ suggest some changes in topological order. The relatively low sensitivity of positron annihilation to the structural relaxation of the amorphous alloy is probably caused by the high density of vacancy-like defects in the alloy.
\end{abstract}

PACS numbers: $75.50 \mathrm{Kj}, 78.70 \mathrm{Bj}$

Positron annihilation (PA) spectroscopy is known as a powerful method for the study of vacancy-like defects in crystalline metals and alloys. Since the half of the seventies a large number of PA studies of amorphous alloys have also been reported. In many of them no changes due to either structural relaxation or to the crystallization were observed. Some of them, on the other hand, show systematic changes in PA spectra due to the processes mentioned but their unambiguous interpretation is still unclear.

In our work, we have investigated the angular correlations of positron annihilation spectra of amorphous alloys $\mathrm{Fe}_{40} \mathrm{Ni}_{40} \mathrm{~B}_{20}$ during their structural relaxation. The aim was to verify the sensitivity of this method to the changes in amorphous $\mathrm{Fe}_{40} \mathrm{Ni}_{40} \mathrm{~B}_{20}$ alloy connected with the annealing out of free volume and possibly with the chemical short-range ordering. As far as $\mathrm{Fe}_{40} \mathrm{Ni}_{40} \mathrm{~B}_{20}$ alloy is concerned, there are some contradictory [1-3]. Balúch et al. [2] found the Ni concentration dependence of the PA characteristics for the amorphous $\mathrm{Fe}_{80-x} \mathrm{Ni}_{x} \mathrm{~B}_{20}$ $(x=10,20,30,40)$ alloys; however, Krištiaková et al. [3] deduced its independence in the same concentration range. Also the changes of PA parameters of the $\mathrm{Fe}_{40} \mathrm{Ni}_{40} \mathrm{~B}_{20}$ alloy due to the structural relaxation and crystallization are rather confusing $[1,3]$. While de Vries et al. [1] state the unchanged positron lifetime after 
crystallization, Krištiaková et al. [3] found the $6 \%$ decrease in this alloy and $23 \%$ increase in the $\mathrm{Fe}_{70} \mathrm{Ni}_{10} \mathrm{~B}_{20}$ alloy and claimed that the annihilation characteristics in amorphous alloys differ from those in the annealed samples in general.

The amorphous alloys of the present study, prepared by the melt spinning technique, were kindly supplied by the Institute of Physics of Electro-Physical Research Center, Slovak Academy of Sciences, Bratislava. From the ribbons $6 \mathrm{~mm}$ wide and $20 \mu \mathrm{m}$ thick, a stack was made consisting of fifteen layers. PA spectra of these samples were taken at room temperature during the interrupted isothermal annealing in $\mathrm{Ar}+\mathrm{H}_{2}$ atmosphere at $473 \mathrm{~K}$ within the time interval from 20 to 200000 min.

The angular correlation PA spectra were measured in the interval from -23 to $+23 \mathrm{mrad}$ in steps of $1.0 \mathrm{mrad}$ by means of a conventional long-slit spectrometer. The width of slits was $1.0 \mathrm{mrad}$ and the number of counts in the peak was about 50000 . The total resolution function was approximated by Gaussian with the full width at half-maximum equal to $1.2 \mathrm{mrad}$ [4]. The spectra were fitted by means of least-squares method to the sum of an inverted parabola and the Gaussian curve convoluted with the total resolution function. In this representation, the parabolic component corresponds to annihilation of positrons with conduction electrons, and Gaussian component to that with ion-core electrons. In the analysis of the angular correlation PA spectra of defected materials, the peak parameter $S$ (defined as a ratio $h / s$ of the height $h$ to the area $s$ of the positron annihilation curve) and the relative intensity $I_{\mathrm{p}}$ of the parabolic component seems to be the most suitable. Trapping of positrons in vacancy-like defects leads to enhanced $S$ and $I_{\mathrm{p}}$ parameters with respect to the defect-free state. Besides the above mentioned model (the sum of an inverted parabola and the Gaussian curve) another models (sums of parabolas and/or of Gaussians) were used, but none of these models gave a better fit. That is why we preferred the peak parameter $S$ independent of the mathematical model used.

The logarithmic time dependence of the $S$ parameter of the amorphous $\mathrm{Fe}_{40} \mathrm{Ni}_{40} \mathrm{~B}_{20}$ is plotted in Fig. 1. Its course is very similar to the annealing time dependence of positron lifetime found in [1]. We can see that there is, within error limits, no change of $S$ after the annealing of amorphous alloy for $20 \times 10^{5} \mathrm{~min}$. at $473 \mathrm{~K}$. Only a local minimum of $S$ corresponding to the annealing time of $2 \times 10^{3}$ min., similar to that one ascertained by the Vries et al. [1] (in [1] denoted as the $B$ point) might suggest some change in topological order. On the other hand, conclusive changes in the ${ }^{57} \mathrm{Fe}$ Mössbauer spectra parameters namely in the average hyperfine induction $B_{\text {ef }}$ at ${ }^{57} \mathrm{Fe}$ nuclei and in the 2 nd to 1st line intensity ratio (reflecting the average magnetization direction), as well as in the static coercivity $H_{C}$ were found in the samples of the same material - amorphous $\mathrm{Fe}_{40} \mathrm{Ni}_{40} \mathrm{~B}_{20}$ alloy and at the same annealing time interval as in the present work [5]. This fact does not confirm the declared sensitivity of positron annihilation to topological and chemical changes due to the annealing of amorphous $\mathrm{Fe}_{40} \mathrm{Ni}_{40} \mathrm{~B}_{20}$ alloys $[1,3]$. The reason, in our opinion, is the high density of vacancy-like defects both in the as-quenched and in the relaxed amorphous states. Since in both these states nearly all positrons are trapped in these defects (due to their affinity to the vacancies) the changes in PA spectra in the course of a sructural relaxation are small. Similarly 


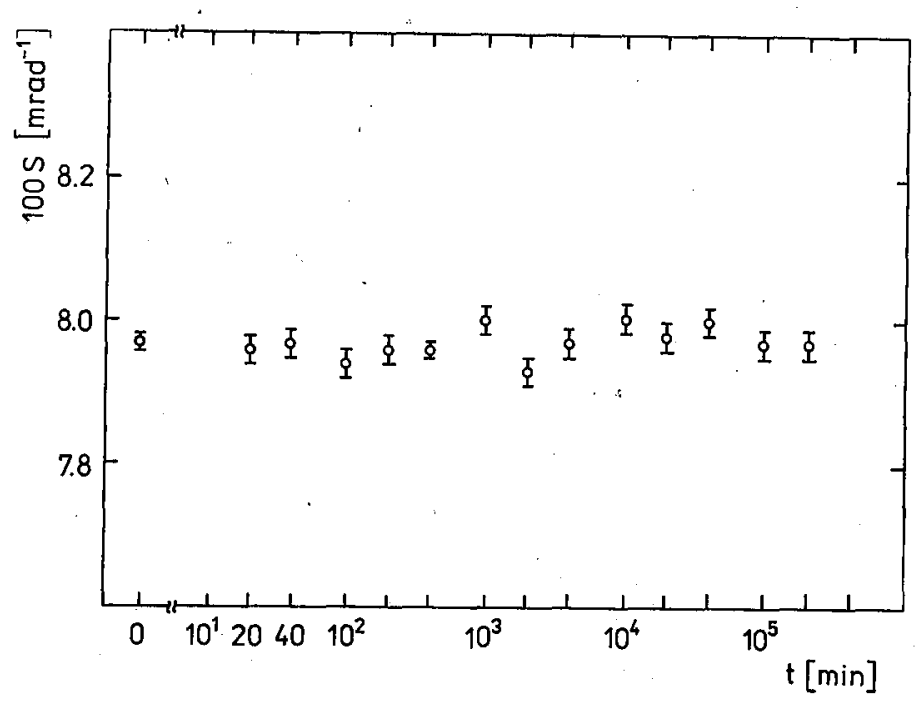

Fig. 1. The dependence of the peak parameter $S$ on the time of isothermal annealing . for amorphous $\mathrm{Fe}_{40} \mathrm{Ni}_{40} \mathrm{~B}_{20}$ alloy.

the high density of trapping centres in numerous grain boundaries of crystalline particles cause inexpressive changes of the PA spectra after the crystallization of amorphous alloys [6].

\section{References}

[1] J. de Vries, G. W. Koebrugge, A. van den Beukel, Scr. Melall. 22, 637 (1988).

[2] S. Balúch, M. Miglierini, R. Gröne, J. Sitek, Phys. Slatus Solidi A 113, K 143 (1989).

[3] K. Krištiaková, G. Tombev, J. Krištiak, Phys. Slalus Solidi A 117, K 5 (1990).

[4] J. Svoboda, M. Šob, Philos. Mag. B 48, 523 (1983).

[5] Y. Jirásková, T. Zemčik, Acla Phys. Pol. A77,695 (1990)

[6] K.P. Gopinathan, J. Madras Univi., B 45, 208 (1982). 